\title{
Adaptive Window Selection for Non-uniform Lighting Image Thresholding
}

\author{
Tapaswini Pattnaik* and Priyadarshi Kanungo $^{+}$ \\ * Department of Electronics and Telecommunication, C. V. Raman Global University, Bhubaneswar, India \\ ${ }^{+}$Department of Electronics and Telecommunication, C. V. Raman Global University, Bhubaneswar,India
}

Received 26th of August2020; accepted 13th of January 2021

\begin{abstract}
Selection of appropriate size of windows or subimages is the most important step for thresholding images with non-uniform lighting. In this paper, a novel criteria function is developed to partition images into different size of sub images appropriate for thresholding. After the partitioning, each subimage is segmented by Otsu's thresholding approaches. The performance of the proposed method is validated on benchmark test images with different degree of uneven lighting. Based on the qualitative and quantitative measures, the proposed method is fully automatic, fast and efficient in comparison to many landmark approaches.
\end{abstract}

Key Words: Image segmentation, Thresholding, Non-uniform Lighting, Local thresholding, Bimodal distribution

\section{Introduction}

Image segmentation is a technique of partitioning an image into homogenous regions. Among all the image segmentation techniques, thresholding is the most popular image segmentation technique for real time application. In thresholding of a two class images, the pixels are divided into two groups at the threshold point. Thresholding techniques [1-4] are broadly classified into two types such as (i) global thresholding and (ii) local thesholding. In global thresholding [5] a single threshold is used to divide all the pixels into two groups. Whereas in local thresholding, the image is divided into sub-images and for each sub-image a threshold is evaluated to binarize the corresponding subimage. Histogram of an image is commonly used for the bi-level or multi-level thresholding evaluation. In histogram based thresholding, Otsu's [6] threshold is one of the landmark global thresholding approach for image binarization. Otsu thresholding is one of the most popular and widely used approach for global thresholding due to its roboustness,simplicity and adaptability. Otsu method is better than most of the other global thresholding because of its high performance on segmenting real images as the calculation of threshold values depends on 1D intensity data. Another advantage of Otsu approach is its simple and strong criteria function [7]. The criteria function maximizing between class variance and minimizing the within class variance produced an efficient segmentation result with low complexity [7-11]. The

Correspondence to: <tapaswini.n@gmail.com>

Recommended for acceptance by Angel D. Sappa

https://doi.org/10.5565/rev/elcvia.1301

ELCVIA ISSN:1577-5097

Published by Computer Vision Center / Universitat Autònoma de Barcelona, Barcelona, Spain 
performance of global thresholding methods decrease in images with non-uniform lighting and poor resolution [12]. In last few decades many literatures [13-22] developed different approaches to binarize images with non-uniform lighting. Niblack [13] used mean and standard deviation to find out the threshold for each pixel in a particular local window. Bradley [16] considered the integral image for the binarization of each pixels. Threshold for each pixel in the integral image is evaluated based on the mean of the neighbouring pixels in a $15 \times 15$ window. Huang's method [17], used the Lorentz information measure as a feature for selection of window size adaptively and then Otsu's thresholding is used to binarize each local window. However, the performance of this method is highly dependent on the initial small size of window. Zheng [18], designed the principle of adaptively adjustment of the local window size. The number of edge pixels in a local window determined the optimal size of the window over a pixel. After getting the optimal window size the range constrained Otsu's thresholding method is applied on window to binarize the center pixel of that window. As this method evaluated threshold for each pixel, the time complexity of this method is very high. High sensitivity to noise and high complexity are the two major drawbacks of this method. Kanungo et al. [19], developed an entropy base window merging and growing process to partition the image into subimages. Further, each subimages is binarized based on the PGA and MMSE based algorithms. The performance of this method is sensitive to the choice of initial window size in both merging and growing process. Zhao [20], evaluated the posterior probability of each center pixel in a $3 \times 3$ or $5 \times 5$ window. Considering the posterior probability and Bayesian criteria the binary map is generated. This method is effective to deal the noise, however the performance of this method is highly depend on the size of the user defined neighbourhood.

Recently Zheng et al. [21], developed an adaptive segmentation based on fuzzy c-means with spatial information (FCMS). The complexity of this method is very high as each pixel is binarized based on the information of different local window. Bogiatzis [23], proposed the local thresholding technique in which pixel is binarized based on fuzzy inclusion and entropy criteria from the fuzzy subset hood of the neighbourhood $M \times N$ of pixel. This method is not automatic as it depends on the optimal choice of the user defined window size. It is observed from the recent literatures that, the performance of all these adaptive thresholding methods is highly sensitive to the partitioning process to generate optimal size of subimages or window size. The objective of the partition process is to find subimages with clearly bimodal gray level or feature distributions.

The review of the literature revels that, the performance of any adaptive thresholding largly depends on the selection of the window size and the bimodal testing criteria function. This motivates us to, develop simple partitioning process which does not need any initial size of window. The second novelty of the proposed method is its non parametric bimodality test criteria function followed by exhaustive experimental evaluation for the selection of threshold for the above criteria function. The criteria function does not need any parameter estimation. Therefore the complexity of the entire process is within the range of real-time application. Experimental results depicts that the proposed method is faster and efficient in comparison to landmark thresholding methods for images with non-uniform lighting. The organisation of this paper is as follows: the formulation of the proposed method (materials and methods) is presented in Section 2. The proposed NMDM algorithm based adaptive image partitioning for thresholding is presented in Section 3. The simulations and discussions are presented in Section 4 followed by the conclusion in Section 5.

\section{Materials and methods}

The major problem of thresholding any images with non-uniform lighting is the development of a criterion function for partitioning the image into optimal size of subimages. To address this problem, in this section a normalized mean difference measure (NMDM) criteria is developed to partition the images with non-uniform lighting images into optimal size of subimages such that the gray level distribution of each sub image satisfy the bimodality property. 


\subsection{Normalized mean difference measure (NMDM)}

In two class images, the histogram of an image represents the gray level distribution. In thresholding, histogram plays a major role to evaluate the optimal threshold. The histogram of a two class image can be model as a two class Gaussian mixture model. For uniform lighting image there is a clear valley between the distributions of object and background class. However the valley between the distributions of object and background vanished in the non-uniform lighting conditions.

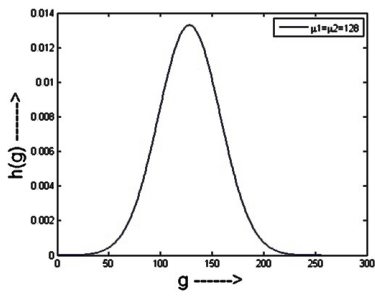

(a)

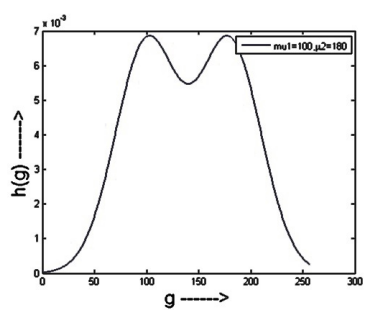

(b)

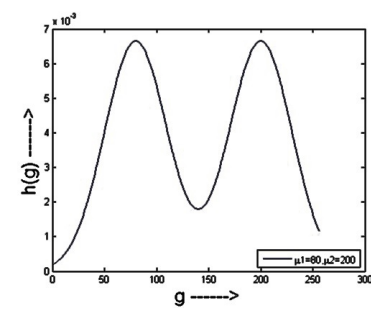

(c)

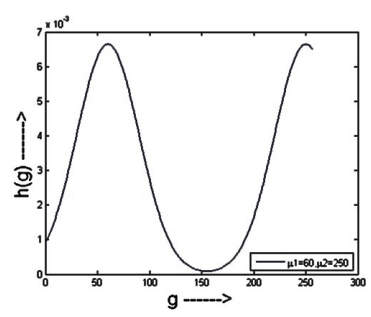

(d)

Figure 1: Simulated two class Gaussian Mixture PDF using (2) $w_{1}=w_{2}=0.5$, and $\sigma_{1}=\sigma_{2}=30$, with four different combination of $\mu_{1}$ and $\mu_{2}$ :(a) $\mu_{1}=\mu_{2}=128$, (b) $\mu_{1}=100$ and $\mu_{2}=180$,(c) $\mu_{1}=80$ and $\mu_{2}=200$, (d) $\mu_{1}=60$ and $\mu_{2}=250$

$$
f(g)=w_{1} P\left(g \mid C_{1}\right)+w_{2} P\left(g \mid C_{2}\right)
$$

Therefore two class image histogram can be modeled as $\mathrm{f}(\mathrm{g})$ in (1). Where, $P\left(g \mid C_{1}\right)$ and $P\left(g \mid C_{2}\right)$ represent Gaussian distribution of class $C_{1}$ and class $C_{2}$ respectively. The gray level distribution $f(g)$ is represented as

$$
f(g)=\frac{w_{1}}{\sqrt{2 \pi \sigma_{1}^{2}}} e^{-} \frac{\left(g-\mu_{1}\right)^{2}}{2 \sigma_{1}^{2}}+\frac{w_{2}}{\sqrt{2 \pi \sigma_{2}^{2}}} e^{-} \frac{\left(g-\mu_{2}\right)^{2}}{2 \sigma_{2}^{2}}
$$

where $w_{1}$ and $w_{2}$ are class probabilities, $\sigma_{1}$ and $\sigma_{2}$ are the standard deviation, $\mu_{1}$ and $\mu_{2}$ are the mean of the class $C_{1}$ and class $C_{2}$ respectively. If the two parameters, $\mu$ and $\sigma$ of $C_{1}$ and $C_{2}$ are equal, then these two distributions are identical distributions with $100 \%$ overlapping. The $\mu_{1}$ and $\mu_{2}$ are the two key parameters which are responsible for the overlapping of the two distributions. Using (1), four different two-class gray level distribution are synthesized and placed in Fig. 1. These four distributions, are synthesized based on $w_{1}=w_{2}=0.5, \sigma_{1}=\sigma_{2}=30$, whereas the $\mu_{1}$ and $\mu_{2}$ are different. Considering $\mu_{1}=\mu_{2}=128$, the distribution is a uni-modal and 100\% overlapping as shown in Fig. 1(a). In Fig. 1(b), the $\mu_{1}=100$ and $\mu_{2}=180$, where there is a reduction in the overlapping of the two distributions in comparison with $\mu_{1}=\mu_{2}$ as shown in Fig. 1(a). Further reduction in overlapping between the two distributions is observed with $\mu_{1}=80$ and $\mu_{2}=200$ as shown in Fig. 1(c). Considering $\mu_{1}=60$ and $\mu_{2}=250$ the overlapping is almost negligible as shown in Fig. 1(d). Let consider the absolute mean difference is $d$, which is defined as $d=\left|\mu_{2}-\mu_{1}\right|$. Keeping $\sigma_{1}$ and $\sigma_{2}$ constant, it is observed that, as $d$ increasing the overlapping between the two distributions is decreasing. Let define the normalized mean difference measure (NMDM) as follows.

$$
d_{\mu}=\frac{d}{\left(G_{\max }-G_{\min }\right)}
$$

where $G_{\max }$ is the maximum and $G_{\min }$ is the minimum gray value of the image. From (3) it is expected that if $d_{\mu}$ is 0 i.e $\mu_{1}=\mu_{2}$ then the maximum overlapping between the two distributions. Similarly if $\mu_{1}=G_{\min }$ and $\mu_{2}=G_{\max }$ then $d_{\mu}=1$ which represents the minimum overlapping between the two distribution. The condition, $d_{\mu}=1$ is an ideal condition for any two class real image. Based on this NMD measure and the standard deviation of the entire image a criterion function is developed in Section 2.2 to measure the bimodal characteristics of the graylevel distribution with acceptable overlapping. 


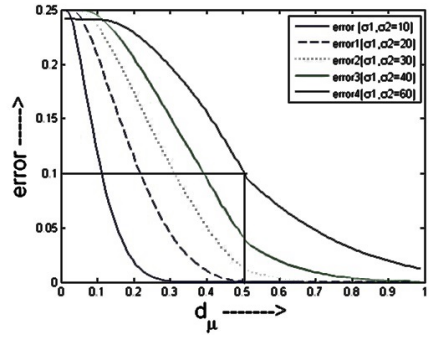

(a)

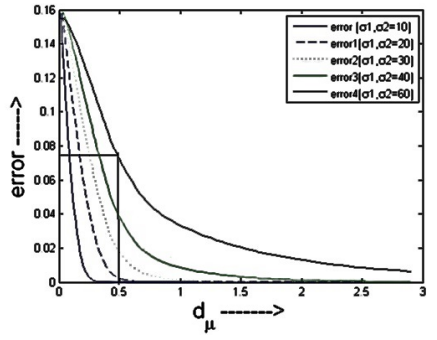

(b)

Figure 2: $P_{E}$ vs $d_{\mu}$ plot considering with different $\sigma_{1}$ and $\sigma_{2}$ (a) $w_{1}=w_{2}=0.5$ and (b) $w_{1}=0.2, w_{2}=0.8$

\subsection{Bimodality Criteria function}

As per Bayes rule, the minimum error partitioning threshold of any two class GMM is the intersection point of these two distributions. Let consider the intersection point of these two distributions in the probability density function(PDF) is at $k^{t h}$ gray level. Then at $k^{t h}$ gray value the equality condition is.

$$
w_{1} P\left(k \mid C_{1}\right)=w_{2} P\left(k \mid C_{2}\right)
$$

The probability of error due to the threshold at $k^{t h}$ gray value can be evaluated as

$$
P_{E}=w_{1} \sum_{g=1}^{k} P\left(g \mid C_{1}\right)+w_{2} \sum_{g=k+1}^{255} P\left(g \mid C_{2}\right)
$$

The probability error, " $P_{E}$ ", increases with the increase in overlapping of class distributions. The effect of $d_{\mu}$ and the standard deviation of the image on $P_{E}$ at different sets of $\left(w_{1}, w_{2}\right)$ and $\left(\sigma_{1}, \sigma_{2}\right)$ are presented in Fig. 2(a) and 2(b). All the five plots in Fig. 2(a) are with equal class probability condition i.e $w_{1}=w_{2}=0.5$. Similarly the five plots in Fig. 2(b) are with unequal class probability condition i.e $w_{1}=0.2$ and $w_{2}=$ 0.8. The five sets of $\left(\sigma_{1}, \sigma_{2}\right)$ considered for simulation are (10 10), (20 20), (30 30), (40 40) and (60 60) for both Fig. 2(a) and Fig. 2(b). It is observed from the considered non uniform light image database that the minimum standard deviation is 25 and the maximum standard deviation is 60 . Therefore we have considered the maximum standard deviation as 60 . The $d_{\mu}$ is varied by varying $\mu_{1}$ and $\mu_{2},\left(\mu_{2} \geq \mu_{1}\right)$, of the $C_{1}$ and $C_{2}$ distributions. Initial value of $\mu_{1}$ and $\mu_{2}$ is considered as 128 and 128 respectively, which results a $d_{\mu}=0$. Further, the $d_{\mu}$ is varied from 0 to 1 with 128 steps by incrementing $\mu_{2}$ by one unit and decrementing $\mu_{1}$ by one unit at each step.It is clearly observed from $P_{E}$ vs $d_{\mu}$ plots in Fig. 2(a) and (b) that for a particular value of $d_{\mu}$ the $P_{E}$ is increasing with increase in standard deviation $\sigma_{1}$ and $\sigma_{2}$. In Fig. 2(a) at $d_{\mu}=0.5$, for $\sigma_{1}=$ $\sigma_{2}=10$ and $\sigma_{1}=\sigma_{2}=20$. It is observed from Fig. 2(a) that $P_{E}$ values for $\sigma_{1}=\sigma_{2}=10$ and $\sigma_{1}=\sigma_{2}=20$ curve at $d_{\mu}=0.5$ are almost zero. It is also observed that, for $\sigma_{1}=\sigma_{2}=30$ the $P_{E}$ is $0.01, \sigma_{1}=\sigma_{2}=40$ the $P_{E}$ is 0.04 , and for $\sigma_{1}=\sigma_{2}=60$ the $P_{E}$ is 0.1. Similarly in Fig. 2(b) there is an increasing trend in the $P_{E}$ with increase in the $\sigma_{1}$ and $\sigma_{2}$ values for a particular $d_{\mu}$. However it is clearly observed that for unequal weighted distribution in Fig. 2(b) the $P_{E}$ is very less than the equal weighted distribution case in Fig. 2(a). For example, considering the $\sigma_{1}=\sigma_{2}=60$, the $P_{E}$ value for $d_{\mu}=0.5$ in Fig. 2(b) is half of the $P_{E}$ value for $d_{\mu}=0.5$ in Fig. 2(a). It is also observed that for $d_{\mu}>0.5$ the $P_{E}$ is almost less than 0.1 for both the conditions in Fig. 2(a) and 2(b). Based on the above observations, it is considered that if $d_{\mu}>0.5$ and the standard deviation of the images $\sigma_{I}<60$, then the gray level distribution of the image depicts the bimodal charaterstics with a maximum overlapping error of $10 \%$. The above criteria function is considered for the adaptive image partitioning for threshold in section 3 . The criteria of bimodality is

$$
C_{I}=\left\{\begin{array}{cc}
1 & \text { if }\left(d_{\mu}>0.5 \& \& \sigma_{I}<60\right) \\
0 & \text { otherwise }
\end{array}\right.
$$


Where $C_{I}=1$ represents the clear bimodality characteristics of an image. The proposed criteria function is based on the parameter $d_{\mu}$ and $\sigma_{I}$. Evaluation of $d_{\mu}$ needs the estimation of parameters $\mu_{1}$ and $\mu_{2}$ of a two class GMM. The parameter estimation using EM algorithm will increase the overall complexity of the proposed adaptive thresholding approach. Therefore simple method using Otsu's thresholding approach is developed to estimate value of $\mu_{1}$ and $\mu_{2}$. The proposed parameter estimation algorithm is as follows.

Algorithm for evaluating the $C_{I}$ :

Step 1 Evaluate the normalized histogram $h(g)$ and Otsu's [6] global threshold ' $T$ ' of the input image I

Step 2. Evaluate the max gray value $\left(G_{\max }\right)$ and minimum gray value $\left(G_{\min }\right)$ of the input at step-1

Step3. Evaluate the $\mu_{1}$ and $\mu_{2}$ as follows

$$
\begin{gathered}
\mu_{1}=\sum_{g=0}^{T} g \times h(g) \\
\text { and } \mu_{2}=\sum_{g=T+1}^{255} g \times h(g)
\end{gathered}
$$

where $g$ is the variable represents the gray value.

Step4. Evaluate the $d_{\mu}=\frac{\left|\mu_{2}-\mu_{1}\right|}{\left(G_{\max }-G_{\min }\right)}$

Step5. Evaluate $\sigma_{I}=\left\{\sum_{g=1}^{255}\left[g-\sum_{g=1}^{255} g \times h(g)\right]^{2} h(g)\right\}^{\frac{1}{2}}$

Step6. Evaluate $C_{I}=\left\{\begin{array}{cc}1 & i f\left(d_{\mu}>0.5 \& \& \sigma_{I}<60\right) \\ 0 & \text { otherwise }\end{array}\right.$

\section{Adaptive Image Partitioning for thresholding}

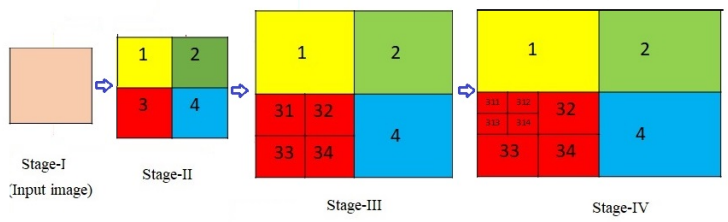

Figure 3: Image Partitioning Process

The proposed partitioning process is a non overlapping partitioning process. To avoid the overpartition and reduce the complexity the minimum size of window is set to $32 \times 32$. As the input images are of different spatial resolution, it is necessary to resize the image in such a way that the image could be partitioned evenly upto $32 \times 32$ windows. The test images collected from database [24] and [25] are less than $256 \times 256$ resolutions and greater than $64 \times 64$ resolutions. Therefore, the images are resized to the higher resolution i.e $256 \times 256$

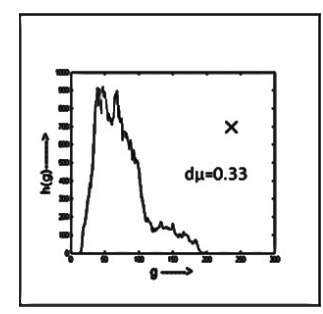

(a)

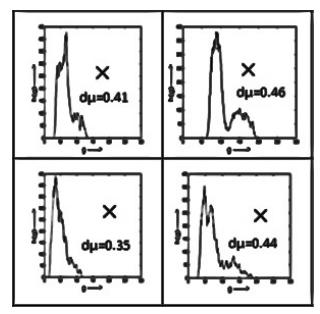

(b)

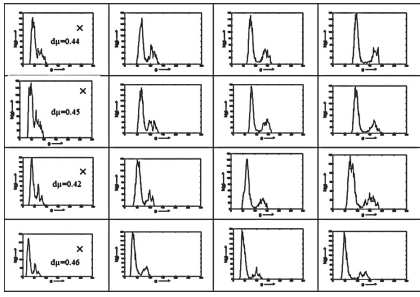

(c)

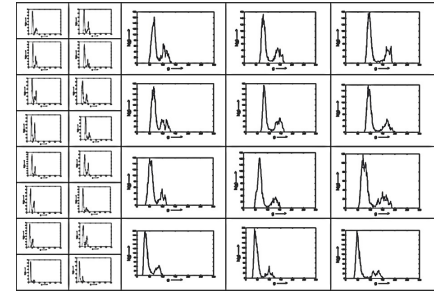

(d)

Figure 4: (a) histogram of input Rice image at stage-I; (b) Four partition at stage-II; (c) 16 partitions in stage-III (d) 28 partition in stage-IV 


\begin{tabular}{|l|l|l|l|l|}
\hline 0.60 & 0.58 & 0.50 & 0.56 & 0.64 \\
\cline { 1 - 2 } 0.57 & 0.54 & & & \\
\cline { 1 - 2 } 0.60 & 0.61 & \multirow{2}{*}{0.50} & 0.58 & 0.56 \\
\cline { 1 - 2 } 0.59 & 0.57 & & & \\
\cline { 1 - 2 } 0.55 & 0.53 & 0.52 & 0.55 & 0.57 \\
\cline { 1 - 2 } 0.59 & 0.54 & & & \\
\cline { 1 - 2 } 0.51 & 0.54 & 0.52 & 0.56 & 0.61 \\
\cline { 1 - 2 } 0.62 & 0.54 & & & \\
\hline
\end{tabular}

(a)

\begin{tabular}{|l|l|l|l|l|}
\hline 14.75 & 18.5 & 22.48 & 29.9 & 24.4 \\
\cline { 1 - 2 } 14.39 & 14.9 & & & \\
\hline 15.3 & 16.5 & 21.08 & 29.83 & 32.4 \\
\cline { 1 - 2 } 13.8 & 16.4 & & & \\
\hline 12.9 & 15.7 & 20.27 & 27.7 & 36.52 \\
\cline { 1 - 2 } 14.12 & 15.27 & & & \\
\hline 12.6 & 16.7 & \multirow{2}{*}{20.23} & 22.77 & 31.82 \\
\cline { 1 - 2 } 10.9 & 11.79 & & & \\
\hline
\end{tabular}

(b)

\begin{tabular}{|l|l|l|l|l|}
\hline 52 & 65 & 85 & 120 & 132 \\
\cline { 1 - 2 } 53 & 66 & & & \\
\hline 54 & 67 & 85 & 109 & 130 \\
\cline { 1 - 2 } 53 & 66 & & & \\
\hline 50 & 61 & 75 & 97 & 114 \\
\cline { 1 - 2 } 46 & 55 & & & \\
\cline { 1 - 2 } 38 & 47 & 57 & 70 & 86 \\
\cline { 1 - 2 } 36 & 38 & & & \\
\hline
\end{tabular}

(c)

Figure 5: (a) $d_{\mu}$ of fig. 4(d) and (b) the corresponding $\operatorname{sigma}(\sigma)$ value (c) Otsu's threshold value for each subimages in Fig. 5(c)

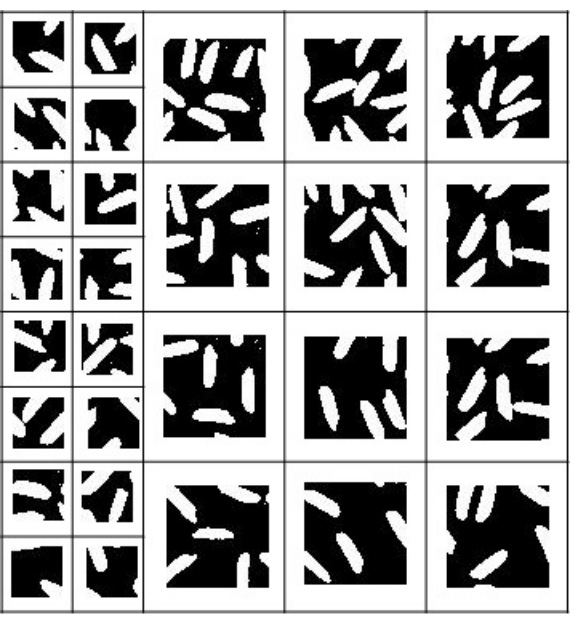

(a)

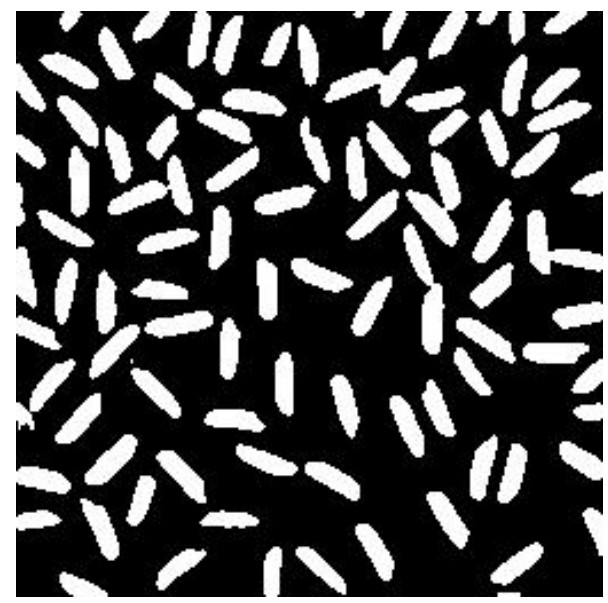

(b)

Figure 6: (a) Segmentation of subimages (b) Final Segmentation results of Rice image

spatial resolution to satisfy the proposed criteria and make the partitioning process more convenient. The proposed image partitioning approach is presented in Fig. 3. In this process, all the images are resized into $256 \times 256$ spatial resolutions. The resizing also reduced the overall non-linear lighting effect of an image. To avoid the overpartition and reduced the complexity the minimum size of window is set to $32 \times 32$. Therefore the proposed partitioning process has maximum of four stages. In (stage-I), the proposed NMD Criteria in (6) is applied on input gray image, If the $C_{I}=1$, then it is binarized using Otsu's thresholding method. Otherwise, the image is partitioned in to four subimages as shown in stage-II. Further the criteria function is applied on each subimages at stage-II. The subimages in the stage-II satisfying the criteria function are not further partitioned in the stage-III. Any subimage in stage-II does not satisfied the criterion function are partitioned into four parts in stage-III. The process described for stage-II applied to the subimages in stage-III and stage IV. The minimum size of subimages in stage-IV is $32 \times 32$ and the maximum size may be $256 \times 256$. If the minimum size of subimages $(32 \times 32)$ resolution at stage-IV does not satisfy $C_{I}=1$ then the threshold value of that subimage is evaluated based on the average of the neighbouring subimages.

The proposed partitioning process and binarization of Rice image demonstrated in Fig. 4. As the histogram(gray level distribution) of Rice image in Fig. 4(a) is not clearly bimodal, the criteria function does not satisfy at stage-I. Therefore the rice image is partitioned into four parts in stage-II. The histogram of these four subimage at stage-II are placed in Fig. 4(b). It is clearly observed that all these histograms are not clearly 
bimodal. Therefore each subimages at stage-II are not satisfying the criteria function. Therefore all these subimages are partitioned into four equal parts at stage-III. There are 16 subimages at stage-III. It is observed form Fig. 4(c) that the histograms of 1st column subimages at stage-III are not following clear bimodality. However the histograms of last three column subimages are exhibiting the bimodality. Therefore, these twelve subimages in last three columns not allowed to partition at the stage-IV. However, each subimage in 1st column of stage-III are divided into equal size subimages at stage-IV as shown in Fig. 4(d). The histograms of all these subimages in 1st and 2nd column of Fig. 4(d) depicts bimodality. In the partitioning process of rice image, all the subimages at stage-IV satisfied the criteria function. Therefore the partitioning process stopped at stage-IV. Otsu's thresholding is applied to each subimages at the stage-IV. The corresponding $d_{\mu}$, the window standard deviation $\sigma_{I}$ and threshold for each subimage are presented in Fig. 5(a), (b) and (c) respectively. The segmented result of each subimage are placed in Fig. 6(a). It is clearly observed from Fig. 6(b) that the proposed method segmented the non-uniform lighted Rice image efficiently.

Table 1: Miss classification error rate $\left(E_{R}\right)$ in $\%$ age

\begin{tabular}{lllllll}
\hline Image & $\begin{array}{l}\text { BRAD } \\
{[16]}\end{array}$ & $\begin{array}{l}\text { FIE } \\
{[23]}\end{array}$ & $\begin{array}{c}\text { LIAW } \\
{[17]}\end{array}$ & $\begin{array}{l}\text { GMM } \\
{[20]}\end{array}$ & $\begin{array}{l}\text { ADRCO } \\
{[18]}\end{array}$ & Proposed \\
\hline Plane & 1.11 & $\mathbf{0 . 7 7}$ & 2.05 & 1.50 & 4.34 & 1.50 \\
Swan & 81.48 & 82.47 & $\mathbf{1 . 5 3}$ & 4.95 & 58.03 & 1.72 \\
DOC & 8.59 & 19.62 & 23.14 & 18.00 & 21.37 & $\mathbf{4 . 1}$ \\
Fish & 11.92 & 20.34 & 3.53 & 8.06 & $\mathbf{3 . 0 7}$ & 3.93 \\
Hex2 & 56.39 & 60.68 & 8.51 & 43.21 & 46.09 & $\mathbf{1 . 3 4}$ \\
Block & 59.64 & 63.21 & 0.51 & 17.15 & 63.7 & $\mathbf{0 . 2 7}$ \\
Sept & 23.68 & 27.2 & $\mathbf{1 . 1 7}$ & 24.56 & 18.11 & 1.41 \\
Rice5 & 29.62 & 62.82 & 8.68 & 15.39 & 36.97 & $\mathbf{1 . 4 1}$ \\
Hex & 51.15 & 52.44 & 8.49 & 27.99 & 31.61 & $\mathbf{1 . 4 6}$ \\
Hand & 48.7 & 53.4 & 3.74 & 15.23 & 30.97 & $\mathbf{2 . 3 1}$ \\
Flower & 18.5 & 21.2 & 7.45 & $\mathbf{0 . 0 1}$ & 18.13 & 3.68 \\
Coin1 & 52.8 & 57.92 & 3.48 & $\mathbf{0 . 2 9}$ & 35.26 & 3.47 \\
86016 & 2.44 & 23.48 & 4.43 & $\mathbf{2 . 1 4}$ & 5.17 & 2.84 \\
311068 & 44.4 & 67.05 & 8.21 & 3.8 & 18.0 & $\mathbf{2 . 8 2}$ \\
Tree & 3.12 & 15.31 & 2.15 & 39.48 & 14.29 & $\mathbf{1 . 8 6}$ \\
pins1 & 6.19 & 12.56 & 2.71 & $\mathbf{1 . 1 5}$ & 4.47 & 2.1 \\
pins2 & 3.55 & 11.47 & 9.97 & 3.87 & 22.2 & $\mathbf{0 . 0 5}$ \\
partree & 15.85 & 27.58 & 15.04 & 1.41 & 3.64 & $\mathbf{0 . 5 0}$ \\
Bandage & 24.89 & 27.58 & 2.00 & $\mathbf{1 . 5 1}$ & 4.34 & 2.17 \\
star & 16.12 & 27.81 & 37.04 & 53.29 & 10.91 & $\mathbf{7 . 7}$ \\
synthetic1 & 67.93 & 63.81 & 9.97 & 5.87 & 51.07 & $\mathbf{0 . 0 5}$ \\
flower1 & 61.38 & 79.89 & 6.25 & 2.15 & 64.09 & $\mathbf{0 . 6}$ \\
Erget & 75.7 & 77.11 & 2.68 & 2.3 & 29.75 & $\mathbf{1 . 9}$ \\
ninety eight & 15.94 & 29.83 & $\mathbf{3 . 0 6}$ & 3.69 & 15.29 & 3.6 \\
coin2 & 29.17 & 40.39 & 1.7 & 1.96 & 16.65 & $\mathbf{1 . 0}$ \\
flower2 & 56.70 & 65.75 & 3.19 & 3.88 & 27.80 & $\mathbf{0 . 8 1}$ \\
fingerprint & 5.29 & 40.03 & $\mathbf{1 . 5}$ & 6.75 & 4.58 & 2.1 \\
Swan2 & 65.92 & 77.16 & 1.9 & 1.5 & 14.02 & $\mathbf{1 . 8}$ \\
\hline AVG $E_{R}$ & 31.3 & 43.17 & 6.57 & 10.23 & 24.02 & $\mathbf{2 . 0 8}$ \\
\hline & & & & & &
\end{tabular}




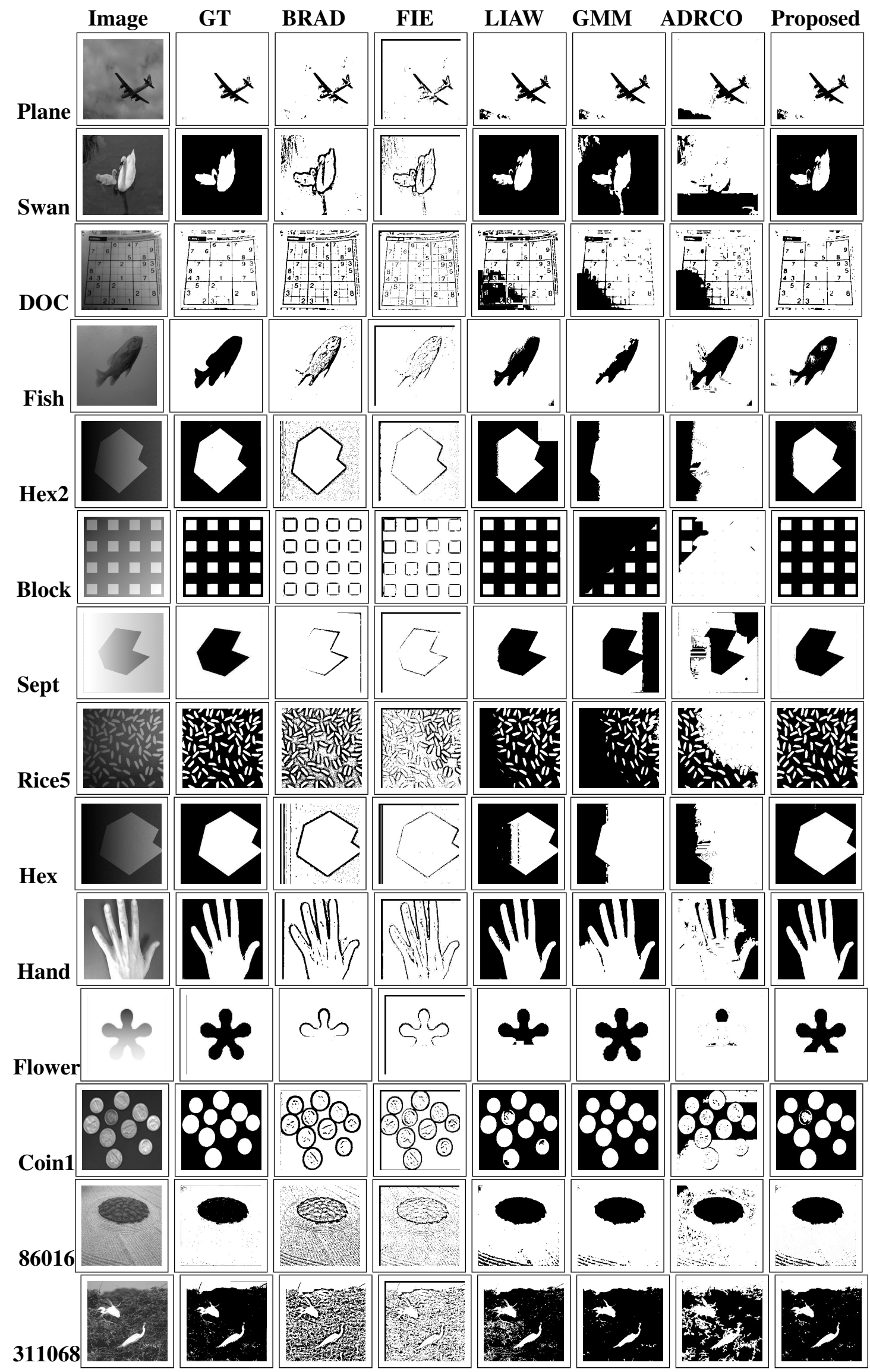

Figure 7: Segmentation results of test images From left column to right column :original test images, Corresponding groundtruth(GT), BRAD [16], FIE [23], LIAW [17], GMM [20], ADRCO [18], and Proposed method 


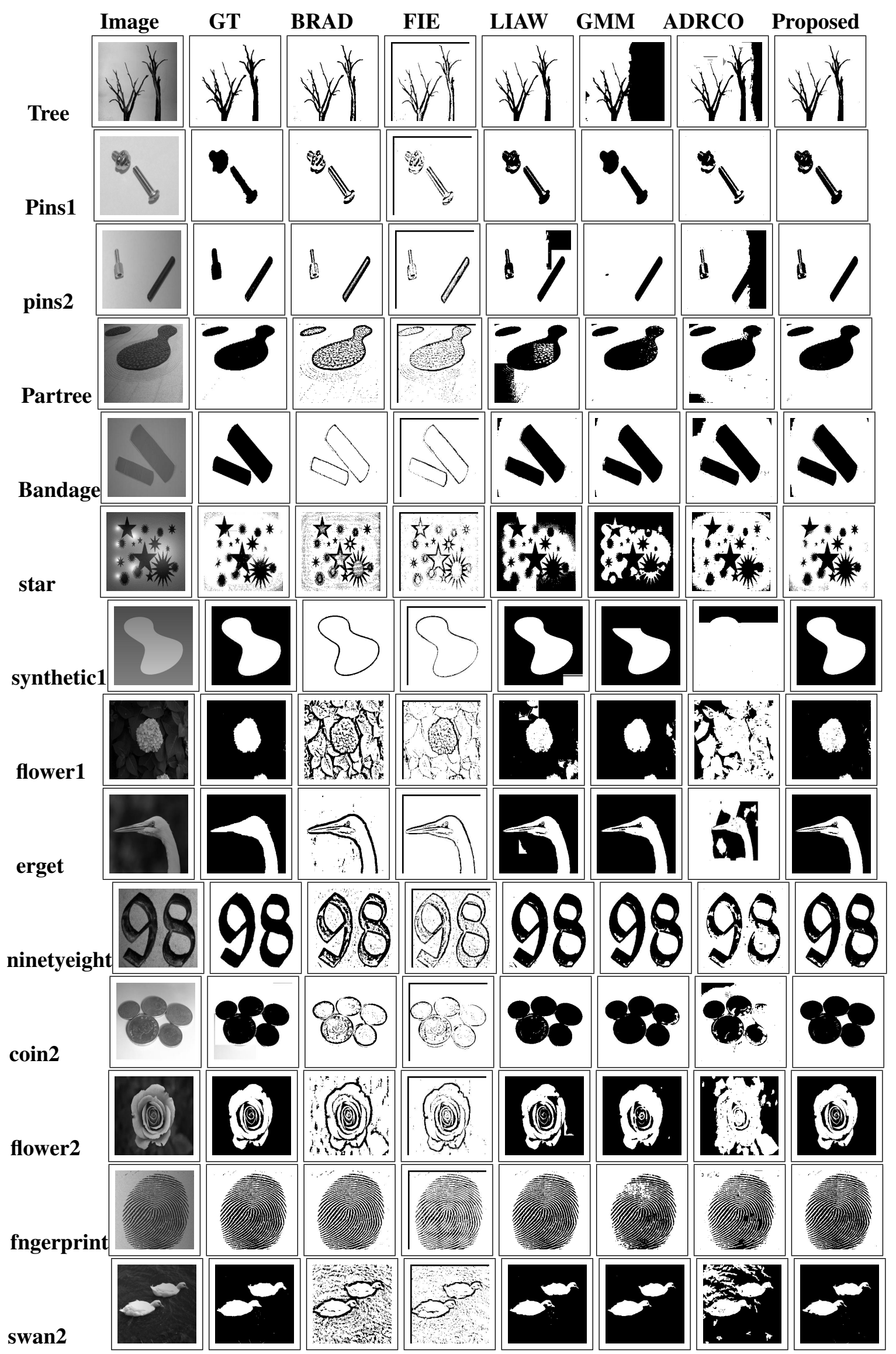

Figure 8: Segmentation results of test images with added illumination From left column to right column :original test images, Corresponding groundtruth(GT), BRAD [16], FIE [23], LIAW [17], GMM [20], ADRCO [18], and Proposed method 
Table 2: Time Complexity $\left(T_{c}\right)$ in Seconds

\begin{tabular}{lllllll}
\hline Image & BRAD & FIE & LIAW & GMM & ADRCO & Proposed \\
& {$[16]$} & {$[23]$} & {$[17]$} & {$[20]$} & {$[18]$} & \\
\hline Plane & 0.12 & 29.37 & 0.023 & 4.03 & 722.4 & 0.28 \\
Swan & 0.13 & 28.7 & 0.025 & 3.64 & 299 & 0.30 \\
Doc & 0.16 & 29.10 & 1.62 & 3.7 & 71.7 & 0.30 \\
Fish & 0.14 & 29.15 & 0.023 & 3.75 & 355.3 & 0.31 \\
Hex2 & 0.13 & 29 & 0.80 & 3.72 & 148.2 & 0.26 \\
Block & 0.16 & 28.9 & 0.08 & 4.02 & 36.12 & 0.40 \\
Sept & 0.18 & 28.97 & 0.037 & 3.7 & 86.6 & 0.41 \\
Rice5 & 0.18 & 28.6 & 0.033 & 3.77 & 26.10 & 0.26 \\
Hex & 0.15 & 30.43 & 0.053 & 3.78 & 210.8 & 0.25 \\
Hand & 0.14 & 29.3 & 0.036 & 3.7 & 94.3 & 0.24 \\
Flower & 0.17 & 28.53 & 0.823 & 3.80 & 600 & 0.42 \\
Coin1 & 0.15 & 29.12 & 0.10 & 4.12 & 62.3 & 0.14 \\
86016 & 0.16 & 29.2 & 0.023 & 4.4 & 40.09 & 0.33 \\
311068 & 0.16 & 29.9 & 0.026 & 3.75 & 69.85 & 0.028 \\
Tree & 0.29 & 29.33 & 0.048 & 3.67 & 106.8 & 0.42 \\
pin1 & 0.15 & 32.02 & 0.043 & 3.62 & 377.5 & 0.62 \\
pin2 & 0.13 & 30.91 & 0.067 & 3.71 & 153.0 & 0.48 \\
partree & 0.18 & 32.71 & 0.044 & 3.57 & 157.9 & 1.37 \\
Bandage & 0.18 & 30.70 & 0.030 & 3.62 & 63.38 & 0.47 \\
star & 0.19 & 27.81 & 0.036 & 3.63 & 9.14 & 0.86 \\
synthetic1 & 0.16 & 31.28 & 0.035 & 3.77 & 78.5 & 0.7 \\
flower1 & 0.13 & 30.25 & 0.044 & 3.74 & 206.8 & 0.25 \\
Erget & 0.13 & 30.42 & 0.035 & 3.76 & 175.0 & 0.31 \\
ninety eight & 0.13 & 29.83 & 0.025 & 4.14 & 4.88 & 0.31 \\
coin2 & 0.13 & 29.71 & 0.037 & 4.06 & 101.5 & 3.34 \\
flower2 & 0.20 & 31.95 & 0.05 & 5.98 & 134.85 & 0.65 \\
fingerprint & 0.24 & 36.48 & 0.032 & 4.53 & 58.72 & 0.68 \\
swan2 & 0.20 & 30.76 & 0.031 & 3.85 & 337.04 & 0.63 \\
\hline Avg $T_{c}$ & $\mathbf{0 . 1 6}$ & 30.08 & $\mathbf{0 . 1 5}$ & 3.75 & 170.9 & $\mathbf{0 . 5 3}$ \\
\hline & & & & & &
\end{tabular}

\section{Simulations and discussions}

The proposed adaptive thresholding is simulated in MatLab 9.5 with an Intel core i5, 4 MB L2 cache, 8GB RAM and $2.4 \mathrm{GHz}$ speed machine. twenty eight test images with different degree of non-uniform lighting are considered for validation of the proposed method. The proposed algorithm is validated with five landmark methods such as BRAD [16], FIE [23], LIAW [17], GMM [20], and ADRCO [18]. The misclassification error rate $\left(E_{R}\right)$ and the time complexity $\left(T_{c}\right)$ are the two measures we considered to measure the strength of the thresholding algorithms. The misclassification error rate is evaluated as follows

$$
E_{R}=\left[1-\frac{\#\left(O_{G T} \bigcap O_{S}\right) \bigcup \#\left(B_{G T} \bigcap B_{S}\right)}{\#\left(O_{G T} \cup B_{G T}\right)}\right] \times 100
$$

where, $O_{G T}$ and $O_{s}$ represent the object region in the GT and segmented image respectively. Similarly $B_{G T}$ and $B_{S}$ represent the background region in the GT and segmented images respectively. The symbol ' \#' represents the cardinality of a set. The time complexity is evaluated based on time elapsed between the starting and to the end of the algorithm. The twenty eight test images are divided into two groups. In the first group, fourteen non 
uniform test images are collected from internet source, Weizmann dataset [24] and Berkeley dataset [25]. The 1 st group test images along with the corresponding binary images generated by different methods are placed in Fig. 7. In the second group, fourteen non-uniform test images are synthesized by adding illumination. Non uniform lighting images collected from weizmann dataset [24]. The 2nd group of test images along with the corresponding binary images generated by different methods are placed in Fig. 8. In Fig. 7 and Fig. 8 the non uniform lighted gray level test images are placed in column 1, the corresponding groundtruth images are placed in column 2 and the corresponding binarized images generated by BRAD [16], FIE [23], LIAW [17], GMM [20], ADRCO [18] and proposed method are placed in column 3 to column 8 respectively. Based on the visual perceptions, it is observed from Fig. 7 and Fig. 8 that the proposed NMD method binarized most of the test images efficiently in comparison to other five methods. It is observed from the Fig. 7 that the performance of the proposed method slightly degrades in the presence of multilevel background like sky in plane image, multilevel object in "coin 1 " test image, textured background in "86016" test image and highly non uniform light conditition as in flower test image. Similarly it is observed from Fig. 8. that the performance of the proposed method slightly degrades in the presence of light reflection from a metal body as in "pins 1 " test image, the low contrast image as in "Bandage" test image. Apart from the above qualitative measures the quantitative measures $E_{R}$ and $T_{c}$ are evaluated for all the methods and tabulated in Table 1 and Table 2. It is observed that in seventeen test images the proposed method has lowest $E_{R}$. The performance of the proposed method in other eleven images is the 2nd lowest $E_{R}$, due to the textured background, background is sky, low contrast image, and reflection of light as discussed in visual result analysis from Fig. 7 and Fig. 8. It is clearly observed that the $E_{R}$ measure for the proposed NMD method is the lowest among all the methods for most of the test images. Therefore the average $E_{R}$ of the proposed approach is $2.08 \%$ which is lowest among all the methods. However for adaptive thresholding methods, ADRCO has the highest time complexity among all the other methods for most of test images. Observing the average time, it is lowest in LIAW Method i.e 0.15 second. However the proposed method has an average computation time of 0.53 , the third lowest computation time with avg $E_{R}$ of $2.08 \%$. In overall, considering qualitative, quantitative and time complexity measures the proposed NMD outperformed the other methods.

\section{Conclusion}

In this paper, an efficient adaptive image partitioning based thresholding technique is developed to segment images with non-uniform lighting. A normalized mean difference measure is formulated to test the bimodality of the image. Otsu's method is used to evaluate the normalized mean difference value of an image. Further the bimodal criteria parameter $C_{I}$ is determined through an exhaustive experimental results. Based on the normalized mean difference criterion function an algorithm is developed to partition the image into optimum number of sub-images. The proposed method has the lowest average misclassification error rate (Avg. $\left.E_{R}\right)$ in comparison with BRAD, FIE, LIAW, GMM and ADRCO. The average CPU time is 0.53 sec/image, which is suitable for real time application. The proposed method outperforms other landmark methods on different bench mark test images with non-uniform lighting in terms of qualitative and quantitative measures. The proposed approach is suitable for gray levels images only. Performance of proposed method slightly decreases on the textured background images and low contrast images affected by illumination. Further to address this issue textural feature can also be gainfully employed which can taken up as a future extension of the paper.

\section{References}

[1] P. K. Sahoo, S. Soltani, A. k. Wong, "A survey of thresholding techniques", Comput. Vision. Graph. Image Process., 41:233-260, 1988, [online]. Available: https://doi.org/10.1016/0734-189X(88)90022-9.

[2] M. Sezgin, B. Sankar, "Survey over image thresholding techniquesand quantitative performance evaluation”, J.Electron Imaging., 13:146-65, 2004, [online]. Available: https://doi.org/10.1117/1.1631315. 
[3] P. Kanungo, P. K. Nanda, and A. Ghosh, "Classification of objects and Background using Parallel Genetic Algorithm Based clustering”, Electronics letters on computer vision and image Analysis., 6(3):42-53, 2007, doi: 10.5565/rev/elcvia.141.

[4] K. S. Deshmukh, "An adaptive Color image segmentation" Electronics letters on computer vision and image analysis., 5(4): 12-23. 2005, doi: 10.5565/rev/elcvia.115

[5] C. Sha, " A roboust Otsu's thresholding method in image segmentation", J. Vis. Commun. Image., 41: 339-351, 2016, doi: 10.1016/j.jvcir.2016.10.013.

[6] N. Otsu, "A threshold selection method from gray-level histograms",IEEE Trans. Syst. Man Cybern., 9: 62-66, 1979, doi: 10.1109/TSMC.1979.4310076.

[7] S. Goh, "performance analysis of image thresholding:Otsu technique",Measurement., 114: 298-307, 2018, doi: 10.1016/j.measurement.2017.09.052.

[8] P. S. Liao, “ A fast algorithm for multilevel thresholding”,Information science and Engineering., 17: 71327. 2001, [online]. Available: https://doi.org/10.1016/j.patrec.2011.01.021

[9] X. Xu, "Characteristic analysis of Otsu threshold and its applications”, Patternrecogn Lett., 32(7): 13-21. 2011, doi: 10.1016/j.patrec.2011.01.021.

[10] H. Cai, Z. Yang, X. Cao, “ A new iterative triclass thresholding technique in image segmentation”,IEEE Trans. Image Process., 23(3): 1038-46. 2014, doi: 10.1109/TIP.2014.2298981.

[11] J. Gong, L. Li, "A fast recursive algorithm for two-dimensional thresholding", IEEE conference on signal processing., 2: 2002, [online]. Available: https://doi.org/10.1016/S0031-3203(97)00043-5.

[12] R. C. Gonzalez, R. E. Woods, Digital Image Processing, 7th ed, Pearson Education, India, 2008.

[13] W. Niblack,An introduction to digital Image Processing, Prentice Hall, Englewood Cliffs, Nj. 1986.

[14] O. Samorodova, "Fast implementation of Niblack Binarization Algorithim for microscopic image segmentation”, Pattern Recognition and Image Analysis., 26: 548-551. 2016, doi: 10.1134/S1054661816030020.

[15] J. Sauvola, "Adaptive document binarisation" Pattern recognition., 33: 225-236. 2000, [online]. Available: https://doi.org/10.1016/S0031-3203(99)00055-2.

[16] D. Bradley, G. Roth, "Adaptive Thresholding using the Integral image" Journal of Graphics Tool., 12: 13-21. 2007, doi: 10.1080/2151237X.2007.10129236.

[17] Q. Huang, W. Gao, W. Cai, "Thresholding technique with adaptive window selection for uneven lighting image" Pattern recognition Letters., 28: 801-08. 2005, doi: 10.1016/j.patrec.2004.09.035.

[18] J. Zheng, D. Zhang, K. Huang, “ Adaptive windowed range constrained otsu method using local information" Journal of electronic Imaging., 25: 013034-1-0134-11. 2016, [online]. Available:https://doi.org/10.1117/1.JEI.25.1.013034.

[19] P. Kanungo, P. K. Nanda, A. Ghosh, “ Parallel genetic algorithm based adaptive thresholding for image segmentation under uneven lighting conditions" IEEE international conference on systems Man and cybernetics.,: 1904-1911. 2010, doi: 10.1109/ICSMC.2010.5642269.

[20] L. Zhao, S. Zheng, W. Vang, “ An image thresholding approach based on gaussian mixture model"Pattern Anal Application., 22: 75-88. 2019, [online]. Available: https://doi.org/10.1007/s10044-018-00769-w. 
[21] J. Zheng, D. Zhang, K. Huang,“ Adaptive image segmentation method based on the fuzzy c-means with spatial information”IET Image Processing., 12(5): 785-792. 2017, doi: 10.1049/iet-ipr.2017.0760.

[22] H. Yazid, H. Arof, “ Gradient based adaptive thresholding” J. Vis. Commun. Image., 32: 926-936. 2013, doi: 10.1016/j.jvcir.2013.06.001.

[23] A. Bogiatzis, B. K. Papadopoulos, “ Local thresholding of degraded or unevenly illuminated documents using fuzzy inclusion and entropy measures" Evolving systems., 10(4): 593-619. 2019, [online]. Available: https://doi.org/10.1007/s12530-018-09262-5.

[24] Alpert, "Weizmann Segmentation Evaluation Database", http://www.wisdom.weizmann.ac.il/ vision/Seg-Evaluation-DB [Access:2007]

[25] Martin, "Berkeley Segmentation Dataset" http://www2.eecs.berkeley.edu/Research/projects/ CS/vision/bsds/ [Access:2007] 